\title{
Lead, Follow or Cooperate? Sequential versus Collusive Payoffs in Symmetric Duopoly Games
}

\author{
Marco A. Marini and Giorgio Rodano \\ Department of Computer, Control and Management Engineering, Sapienza University of Rome, Via Ariosto 25, 00185 Rome, Italy
}

Correspondence should be addressed to Marco A. Marini; marini@dis.uniromal.it

Received 18 August 2013; Accepted 15 September 2013

Academic Editors: M. E. Kandil and M. Tsionas

Copyright (C) 2013 M. A. Marini and G. Rodano. This is an open access article distributed under the Creative Commons Attribution License, which permits unrestricted use, distribution, and reproduction in any medium, provided the original work is properly cited.

\begin{abstract}
In many strategic settings comparing the payoffs obtained by players under full cooperation to those obtainable at a sequential (Stackelberg) equilibrium can be crucial to determine the outcome of the game. This happens, for instance, in repeated games in which players can break cooperation by acting sequentially, as well as in merger games in which firms are allowed to sequence their actions. Despite the relevance of these and other applications, no full-fledged comparisons between collusive and sequential payoffs have been performed so far. In this paper we show that even in symmetric duopoly games the ranking of cooperative and sequential payoffs can be extremely variable, particularly when the usual linear demand assumption is relaxed. Not surprisingly, the degree of strategic complementarity and substitutability of players' actions (and, hence, the slope of their best replies) appears decisive to determine the ranking of collusive and sequential payoffs. Some applications to endogenous timing are discussed.
\end{abstract}

\section{Introduction}

Standard game-theoretic settings dealing with the emergence of cooperation usually weight the stream of players' payoffs colluding a finite or infinite number of periods to those obtained by defecting one period and then playing simultaneously à la Nash (noncooperatively) afterward. The possibility that players defect from the collusive outcome as leaders or followers in every stage game is usually not considered. An exception to this approach is contained, for instance, in Mouraviev and Rey [1], who study the role of price (or quantity) leadership in facilitating firm collusion in an infinitely repeated setting. They show that, under price competition and, to a much lesser extent, under quantity competition, the possibility that players sequence their actions in every stage may help to sustain collusion. This happens because the presence of a deviating leader makes it easy for the follower to punish such defection.

In general, the focus on the link between timing and collusion is not entirely new within the economic literature. For instance, in some classical contributions on cartels and mergers under oligopoly, colluding firms are assumed to act as Stackelberg leaders [2-6]. Moreover, a few recent papers on mergers and R\&D agreements consider different timing structures, where groups of firms can either act as leaders or followers (e.g., [7-10]). In these and other potentially interesting economic applications, it is crucial to compare the payoff under collusion to those under noncooperative sequential play to determine the outcome of the game.

While the literature comparing leader and follower's (as well as simultaneous Nash) payoffs has a long-standing tradition (see [11, 12] or [13] for references), the number of papers that compares collusive and sequential outcomes even in a simple duopoly framework appears, at the best, scant. If, on the one hand, it has been proved that in regular symmetric duopoly games with single-valued best-replies and monotone payoffs on rivals' actions (denoted monotone spillovers), when actions are strategic complements, the follower's payoff dominates which of the leader and the opposite holds under strategic substitutes (e.g., [13-15]. In particular, by relaxing the assumption of complements or substitutes actions, [13] proves that either the leader's payoff dominates that of the follower (which is also dominated by the simultaneous Nash) or, in turn, is dominated by the follower's. Dowrick [16], Amir [17], Amir and Grilo [18], Amir et al. [15], Currarini and Marini [19, 20], and Von Stengel and Zamir [21] all present 
various leader-follower payoff comparisons between single players or coalitions. On the other hand, the relationship between fully cooperative and sequential payoffs has been scarcely explored. To the best of our knowledge, in an oligopoly setup with quantities acting as strategic substitutes, Levin $[6,22]$ has provided a comparison between prices, quantities, and social welfare in Cournot, Stackelberg, and monopoly equilibria. In a symmetric setting, Figuières et al. [23] have considered, in turn, symmetric conjectural, simultaneous Nash and Pareto-optimal interior equilibria. However, their model assumes symmetric conjectures for players, and, therefore, their main results do not apply to the analysis of a Stackelberg equilibrium, which is naturally asymmetric.

Our paper considers a class of symmetric duopoly games and shows that the ranking of cooperative and sequential payoffs can be extremely variable. In particular, when actions are strategic substitutes and spillovers negative, it is shown that, rather surprisingly, the leader can earn in some cases a higher profit than the equal-split cooperative (efficient) payoff, and this occurs, in particular, when players' best replies are very (and negatively) sloped. This is because the leader can exploit in full her first-mover advantage when the follower strongly reduces his strategy in response. Exactly the reverse occurs in a game with strategic substitutes and positive spillovers. Here the leader can do better than under symmetric collusion when the follower is not very reactive. A similar but opposite reasoning applies to the case of strategic complements: with negative spillovers, equal-split cooperative payoffs are lower than follower's payoff when best replies are very flat, and the opposite under positive spillovers. In this paper we also provide a taxonomy of all feasible ranking of payoffs and actions of players arising in simultaneous, sequential, and collusive equilibria when actions are either strategic complements or substitutes.

At the end of the paper we discuss some possible implications of our results. To this aim, we introduce an elementary endogenous timing game in which players can decide the timing of their cooperative or noncooperative strategies. Thus, we show that when binding agreements among players are allowed, intertemporal cooperation is in general more vulnerable to defection than cooperation occurring at just one stage.

The paper is organized as follows. The next section introduces the basic setup of the paper. Section 3 presents some results concerning players' equilibrium strategies and payoffs. To understand the forces at work, some simple examples of price and quantity competition are briefly presented. Section 4 applies some of the paper results to the issue of endogenous intertemporal cooperation Section 5 concludes.

\section{The Setup}

We assume two players $i=1,2$ with identical strategy sets $X_{i}=X \subset \mathbb{R}_{+}$and symmetric payoffs $u_{i}\left(x_{i}, x_{j}\right): X^{2} \rightarrow \mathbb{R}$, that is, such that, for every profile $\left(x_{i}, x_{j}\right) \in X^{2}, u_{i}\left(x_{i}, x_{j}\right)=$ $u_{j}\left(x_{j}, x_{i}\right)$. We restrict payoffs to be either strictly positive or strictly negatively monotone on rival's strategy. We talk, in turn, of positive (PS) and negative spillovers (NS). Moreover, players' actions are defined strategic complements (substitutes) if and only if, for every $i=1,2$ and $j \neq i$, the payoff $u_{i}\left(x_{i}, x_{j}\right)$ exhibits increasing (decreasing) differences in $\left(x_{i}, x_{j}\right) \in X^{2}$, that is, $u_{i}\left(x_{i}, x_{j}^{\prime}\right)-u_{i}\left(x_{i}, x_{j}^{\prime \prime}\right) \geq 0(\leq 0)$ for every $x_{j}^{\prime \prime}, x_{j}^{\prime} \in X$ with $x_{j}^{\prime \prime}>x_{j}^{\prime}$. It is well known that, with smooth players' payoffs, increasing (decreasing) differences hold if and only if $\partial^{2} u_{i} /\left(\partial x_{i} \partial x_{j}\right) \geq(\leq) 0$ [24]. We will assume, in what follows, strictly increasing or strictly decreasing differences of players' payoffs, which, in turn, imply strictly increasing or strictly decreasing players' best-replies.

We can now define the behaviour of players in the different scenarios. Under perfect collusion, players are assumed to set cooperatively their strategy profile

$$
\mathbf{x}^{c}=\left(x_{i}^{c}, x_{j}^{c}\right),
$$

where, for every $i$ th player,

$$
x_{i}^{c}=\arg \max _{x_{i}} \sum_{i=1,2} u_{i}\left(x_{i}, x_{j}\right) .
$$

The above formulation implies that the two players possess transferable utilities. No side payments are explicitly allowed and, therefore, payoff allocation only depends on the strategies played by the two players at the (efficient) profile (1). In the following analysis we will assume a symmetric cooperative solution, namely, $x_{i}^{c}=x_{j}^{c}=x^{c}$ and, therefore, $u_{i}\left(x^{c}, x^{c}\right)=u_{j}\left(x^{c}, x^{c}\right)$. We show in Appendix that, for a large class of games (such as games with strict quasiconcave payoff functions and convex strategy sets), the cooperative solution is symmetric. (See also $[25,26]$.) Depending on the model applications such solution can be interpreted, in turn, as the formation of a merger (or a cartel), or as tacit collusion. When players are assumed to move simultaneously and noncooperatively, they play à la Nash, and the equilibrium strategy profile is

$$
\mathbf{x}^{n}=\left(x_{i}^{n}, x_{j}^{n}\right),
$$

where, for every $i, j=1,2$ and $j \neq i$

$$
x_{i}^{n}=\arg \max _{x_{i}} u_{i}\left(x_{i}, x_{j}^{n}\right) .
$$

By symmetry, if the Nash equilibrium $\mathbf{x}^{n}$ of the duopoly game is unique, it must be symmetric: $x_{i}^{n}=x_{j}^{n}=x^{n}$. Finally, when players act sequentially, we assume that the relevant equilibrium concept is the Stackelberg (subgame perfect Nash) equilibrium, that is, the profile

$$
\mathbf{x}^{s}=\left(x_{i}^{s}, r_{j}\left(x_{i}^{s}\right)\right),
$$

where, for the leader (henceforth player $i$ )

$$
x_{i}^{s}=\arg \max _{x_{\mathrm{i}}} u_{i}\left(x_{i}, r_{j}\left(x_{i}\right)\right)
$$

and, for the follower (henceforth player $j$ ), $r_{j}: X \rightarrow X$ such that

$$
r_{j}\left(x_{i}\right)=\arg \max _{x_{j}} u_{j}\left(x_{i}, x_{j}\right) .
$$


Note that if players' payoffs are continuous and strictly quasiconcave, best replies are continuous and single valued. In addition, if players' strategy sets are compact and convex, Brower's fixed-point theorem ensures the existence of a Nash equilibrium $\mathbf{x}^{n}$ of the simultaneous move game. The existence of a Stackelberg equilibrium $\mathbf{x}^{s}$ requires both the continuity of the leader's payoff on her action as well as a follower's continuous best reply, implying that the leader faces a continuous maximization problem over a compact set. In turn, the existence of the cooperative equilibrium $\mathbf{x}^{c}$ does not pose particular problems when players' payoffs are continuous and strategy sets compact. The uniqueness of equilibria (1)-(5) is, in general, a more demanding property. When not specifically stated differently, we will assume it.

\section{Main Results}

It is well known that, in symmetric duopoly games with monotone spillovers and single-valued best replies, if actions are strategic substitutes (and best-replies decreasing), players' payoffs under equilibria ((3)-(5)) respect the following inequality:

$$
u^{L} \geq u^{N} \geq u^{F},
$$

where $u^{N}$ indicates a player's payoff at the simultaneous Nash equilibrium (3), and $u^{L}$ and $u^{F}$ denote, respectively, the leader and the follower's payoffs at the subgame perfect Nash (Stackelberg) equilibrium (5). Since we have assumed strictly increasing or strictly decreasing players' best-replies and monotone spillovers, we can exclude the trivial interior equilibria in which $\mathbf{x}^{n}=\mathbf{x}^{s}$ and, therefore, $u^{L}=u^{N}=u^{F}$. As a result, (8) becomes

$$
u^{L}>u^{N}>u^{F} .
$$

When, conversely, players' actions are strategic complements (and best-replies increasing), it is obtained that

$$
u^{F}>u^{L}>u^{N} \text {. }
$$

This means that, when (9) holds, since all players prefer to lead and none to follow, in the endogenous timing game $a$ la Hamilton and Slutsky [27] - known as extensive form game with observable delay - where two players declare simultaneously their intention to play early or late a given strategic game, there exists a unique pure subgame perfect Nash equilibrium in which players end up playing simultaneously. If, conversely, (10) holds, both sequential (Stackelberg) equilibria, with either order of play among players, are supported as subgame perfect Nash equilibrium of the extended game.

In what follows, some of the results presented do not strictly require the monotonicity of players' best-replies (implied, in turn, by the property of increasing or decreasing differences of players' payoffs). However, for simplicity, our main results are characterized for two well-known classes of duopoly games in which actions are, in turn, strategic complements and substitutes. The first result is rather trivial, and it is simply based on (8)-(10) and on the Pareto-efficiency of the symmetric collusive outcome.
Proposition 1. In all symmetric duopoly games in which players' actions are strategic complements (substitutes), the symmetric collusive payoff of every player must be higher than leader's (follower's) equilibrium payoff at the sequential game, namely, $u^{C}>u^{L}\left(u^{C}>u^{F}\right)$.

Proof. Suppose by contradiction that, if duopoly game actions are strategic complements, $u^{C} \leq u^{L}$. By (10), $u^{C} \leq$ $u^{L}<u^{F}$ and using symmetry, $2 u^{C}<u^{L}+u^{F}$, contradicting the efficiency of the cooperative strategy profile $\mathbf{x}^{c}$. The same occurs when actions are strategic substitutes and $u^{\mathrm{C}} \leq$ $u^{F}$.

Corollary 2. In all symmetric duopoly games in which players' actions are strategic complements (substitutes), the following payoff ranking arises: $u^{C}>u^{L}>u^{N}\left(u^{C} \geq u^{N}>u^{F}\right)$.

Proof. This is obtained by combining symmetry, the results of Proposition 1, and the efficiency of the cooperative allocation.

However, to obtain a complete ranking of players' payoffs, it remains to be ascertained if, in turn, $u^{C} \geq u^{F}$ or $u^{F}>u^{C}$ for actions that are strategic complements and $u^{C} \geq u^{L}$ or $u^{L}>u^{C}$ for actions that are strategic substitutes. As a first step we focus our attention on games with strategic complements.

3.1. Games with Strategic Complements. We present here some results on the relationship between players' equilibrium strategies and their payoffs when actions are strategic complements. The next result provides a sufficient condition for the follower's payoff to overcome the symmetric collusive payoff.

Proposition 3. In all symmetric duopoly games with strategic complements and negative (NS) (positive (PS)) spillovers, if at the Stackelberg equilibrium the leader plays a strategy that is lower (higher) than that played under collusion, namely, $x_{i}^{s}<$ $x^{c}\left(x_{i}^{s}>x^{c}\right)$, the following payoff ranking arises: $u^{F}>u^{C}>$ $u^{L}>u^{N}$.

Proof. If, at the Stackelberg equilibrium, player $i(i=1,2)$ as leader plays a strategy such as $x_{i}^{s}<x^{c}$ (under NS) and $x_{i}^{s}>x^{c}$ (under PS), thus, for the follower

$$
u_{j}\left(x_{i}^{s}, r_{j}\left(x_{i}^{s}\right)\right) \geq u_{j}\left(x_{i}^{s}, x^{c}\right)>u_{j}\left(x^{c}, x^{c}\right),
$$

where the first inequality stems from the Nash property of best-reply $r_{j}(\cdot)$ and the second from the property of monotone spillovers and the fact that $x^{c}>x_{i}^{s}$ under NS and $x^{c}<x_{i}^{s}$ under PS. Thus, by symmetry, $u_{j}\left(x_{i}^{s}, r_{j}\left(x_{i}^{s}\right)\right)=u^{F}$ and $u_{j}\left(x^{c}, x^{c}\right)=u^{C}$ and, by Proposition 1 , it follows that $u^{F}>$ $u^{C}>u^{L}>u^{N}$. Example 4 below shows that the condition is only sufficient and, by no means, necessary for the result. Figures 1 and 2 illustrate the two cases of proposition under NS and PS.

Example 4 (Cournot with strategic complements). Let two firms face an inverse demand function $P(Q)=(1+Q)^{-b}$, 


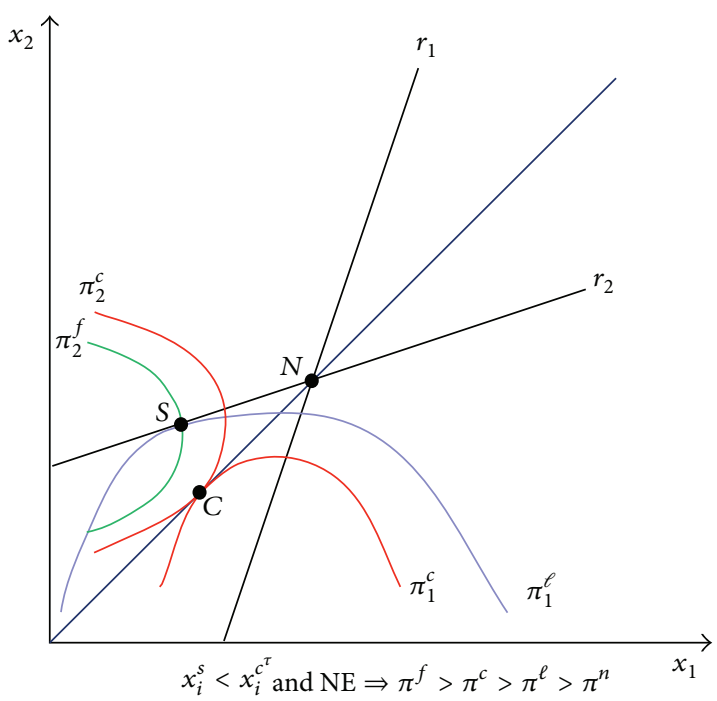

Figure 1: Duopoly game with strategic complements and negative externalities-red: coop. isoprofits; blue: leader's isoprofit; green: follower's isoprofit.

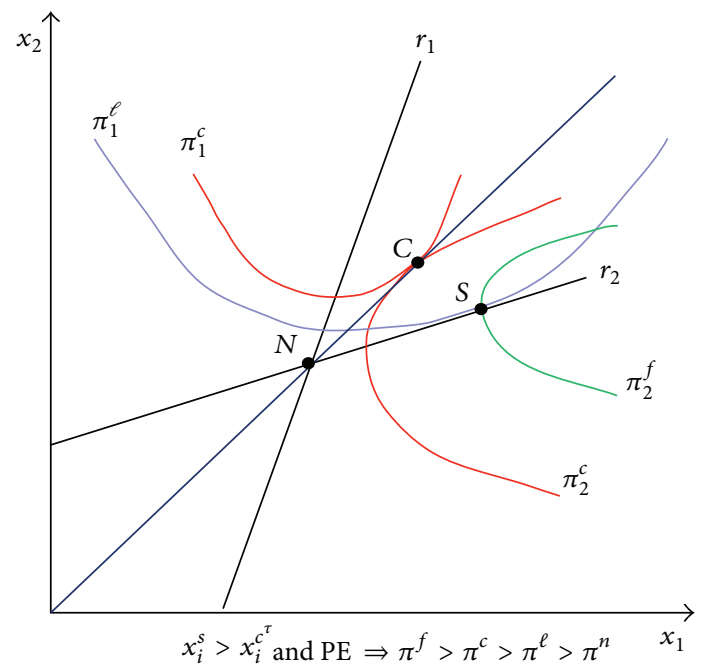

FIGURE 2: Duopoly Game with strategic complements and positive externalities-red: coop. isoprofits; blue: leader's isoprofit; green: follower's isoprofit.

where $Q=\left(q_{1}+q_{2}\right)$ and $b>1$, and, for simplicity, production costs set to zero. Every firm payoff is $u_{i}\left(q_{i}, Q\right)=$ $(1+Q)^{-b} q_{i}, i=1,2$, and it is easy to see that spillovers are negative and quantities act as strategic complements, yielding increasing best replies. Simple computations show that the equilibrium quantities are, respectively, $q^{n}=1 /(b-2)$, $q_{i}^{s}=1 /(b-1), q_{j}^{s}=b /(b-1)^{2}$, and $q^{c}=1 /(2(b-1))$. Equilibrium payoffs are $u^{N}=(b-2)^{-1}(b /(b-2))^{-b}, u^{L}=$ $(b-1)^{-1}\left(b^{2} /(b-1)^{2}\right)^{-b}, u^{F}=(b-1)^{-2} b\left(b^{2} /(b-1)^{2}\right)^{-b}$, and $u^{C}=(2 b-2)^{-1}(b /(b-1))^{-b}$. It is straightforward to see that, for $b>2, q^{n}>q_{j}^{s}>q_{i}^{s}>q^{c}$ and $u^{C} \geq u^{F}>u^{L}>u^{N}$. Figure 4 illustrates this case. For $1<b<2$, there is a switch in the

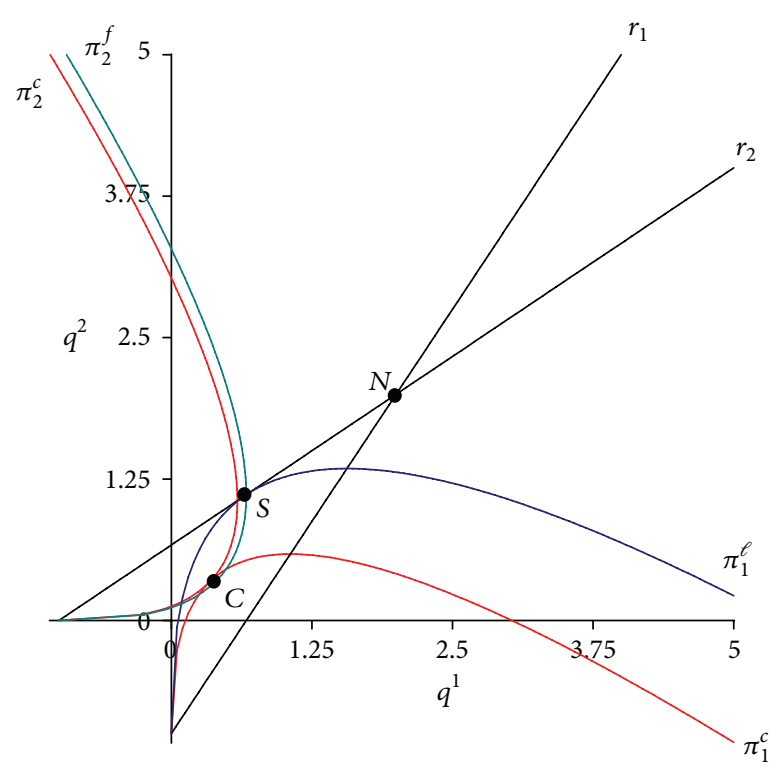

Figure 3: Numerical example (Cournot with strategic complements)—case $b=2.5$. Red: coop. isoprofits; blue: leader's isoprofit; green: follower's isoprofit.

ranking of payoffs and $u^{F}>u^{C}>u^{L}>u^{N}$. Note that, for $1<b \leq 2$, firm's best replies are not contraction and, for this range of parameters, a simultaneous (Cournot) pure strategy Nash equilibrium does not exist. However, since $q_{i}^{s}=1 /(b-1)$ and $q^{c}=1 /(2(b-1)), q^{c}<q_{i}^{s}$ holds for any level of $b$. This proves that the condition of Proposition 3 is only sufficient and not necessary for inequality $u^{F}>u^{C}$ to arise. This is illustrated in Figure 4. As in the standard Cournot duopoly, lower isoprofit curves for firm 1 (or isoprofit curves more on the left for firm 2) correspond to higher profit levels.

The next proposition illustrates the relation between leader's and follower's strategy at any Stackelberg equilibrium in which the follower obtains a higher payoff than under collusion. Together with Proposition 3, it helps to see that, when strategies are complements (and best-replies increasing), a Stackelberg equilibrium always lies below the 45-degree line under negative externalities and above the 45-degree line under positive spillovers (see Figures 2, 3, and 4).

Proposition 5. In all symmetric duopoly games with strategic complements, if the follower's payoff at the Stackelberg equilibrium is higher than the payoff obtained at the symmetric cooperative equilibrium, namely, $u^{F}>u^{C}$, the strategy level of the follower is higher (lower) than the leader's under negative (positive) externalities, namely, $x_{j}^{s}>x_{i}^{s}$ under NS and $x_{j}^{s}<x_{i}^{s}$ under PS, where $x_{j}^{s}=r_{j}\left(x_{i}^{s}\right)$.

Proof. Assume that $x_{j}^{s}<x_{i}^{s}$ under NS and $x_{j}^{s}>x_{i}^{s}$ under PS. Thus,

$$
u_{j}\left(r_{j}\left(x_{i}^{s}\right), r_{j}\left(x_{i}^{s}\right)\right)>u_{j}\left(x_{i}^{s}, r_{j}\left(x_{i}^{s}\right)\right)>u_{j}\left(x^{c}, x^{c}\right) \text {, }
$$




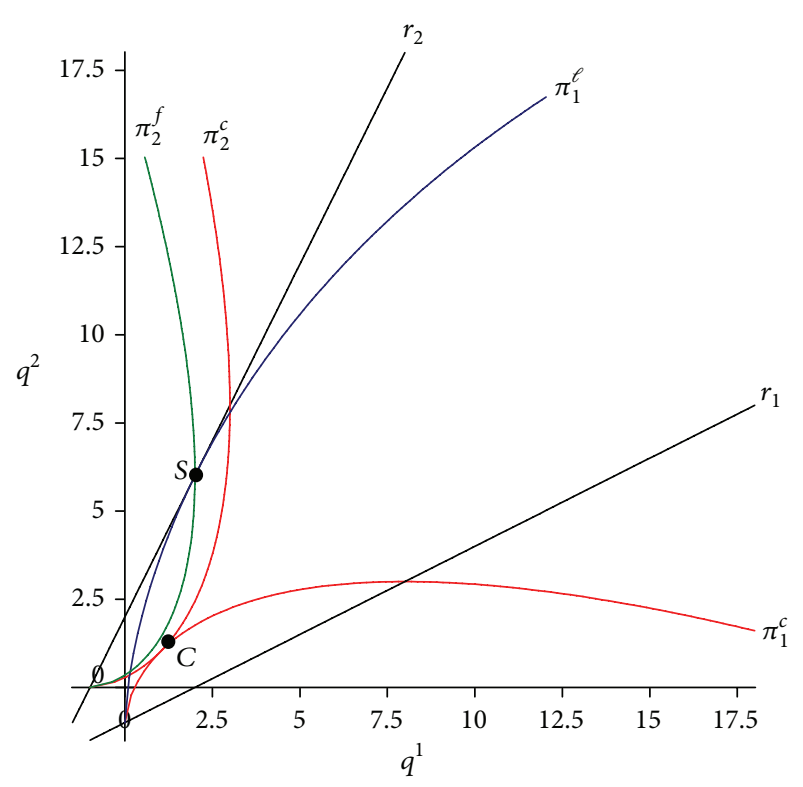

FIgURE 4: Numerical example (Cournot with strategic complements)—case $b=1.5$. Red: coop. isoprofits; blue: leader's isoprofit; green: follower's isoprofit.

where the first inequality is due to monotone spillovers and the second to the fact that $u^{F}>u^{C}$. Since by symmetry $u_{j}(x)=u_{i}(x)$ for any strategy profile $x$ in which $x_{i}^{n}=x_{j}^{n}$,

$$
\begin{gathered}
u_{i}\left(r_{j}\left(x_{i}^{s}\right), r_{j}\left(x_{i}^{s}\right)\right)+u_{j}\left(r_{j}\left(x_{i}^{s}\right), r_{j}\left(x_{i}^{s}\right)\right) \\
>u_{i}\left(x^{c}, x^{c}\right)+u_{j}\left(x^{c}, x^{c}\right)
\end{gathered}
$$

which contradicts the efficiency of $x^{c}$.

The next proposition characterizes instead the order of strategies in the standard case in which the collusive agreement yields a higher payoff for a player than playing as a follower the sequential game. Again, to make things simple, we assume that Stackelberg and simultaneous Nash equilibrium differ, that is, $\mathbf{x}^{s} \neq \mathbf{x}^{n}$.

Proposition 6. In all symmetric duopoly games with strategic complements, if the symmetric collusive payoff is at least as high as the follower's payoff at the Stackelberg equilibrium; namely, $u^{C} \geq u^{F}$, the following ranking between equilibrium strategies arises: $x^{n}>x_{j}^{s}>x_{i}^{s} \geq x^{c}$ under NS and $x^{c} \geq x_{i}^{s}>x_{j}^{s}>x^{n}$ under PS, where $x_{j}^{s}=r_{j}\left(x_{i}^{s}\right)$, for $i, j=1,2$ and $j \neq i$.

Proof. The fact that $x_{i}^{s} \geq x^{c}$ under negative spillovers (NS) and $x_{i}^{s} \leq x^{c}$ under positive spillovers (PS) whenever $u^{C} \geq u^{F}$ is directly implied by Proposition 3 . The remaining inequalities are standard (see, for instance, Amir et al., [28]): taking player $i$ in the role of leader,

$$
u_{i}\left(x_{i}^{s}, r_{j}\left(x_{i}^{s}\right)\right)>u_{i}\left(x^{n}, x^{n}\right) \geq u_{i}\left(x_{i}^{s}, x^{n}\right),
$$

where the first inequality is due to the fact that $\mathbf{x}^{s} \neq \mathbf{x}^{n}$ and the second by the Nash property of $\mathbf{x}^{n}$. Thus, (14) directly implies that $r_{j}\left(x_{i}^{s}\right)=x_{j}^{s}<x^{n}$ under NS and $r_{j}\left(x_{i}^{s}\right)=x_{j}^{s}>x^{n}$ under PS. Given that players' actions are strategic complements (and best-replies increasing), it also follows that $x_{i}^{s}<x^{n}$ under NS and $x_{i}^{s}>x_{i}^{n}$ under PS, since both Stackelberg and simultaneous Nash profiles lie along the increasing follower's best reply. Finally, since the simultaneous Nash equilibrium is symmetric and lies on the 45-degree line, the latter inequalities $\left(x_{i}^{s}<x^{n}\right.$ under NS and $x_{i}^{s}>x_{i}^{n}$ under PS) forcefully imply that $x^{s}$ lies above the 45-degree line under NS and below this line under PS. Hence, $x_{j}^{s}>x_{i}^{s}$ under NS and $x_{j}^{s}<x_{i}^{s}$ under PS, and this concludes the proof.

So far, we have shown that, in duopoly games with strategic complements, two main strategy-payoff equilibrium combinations are possible in the nontrivial case in which $\mathbf{x}^{s} \neq \mathbf{x}^{n}$.

Proposition 7. In all symmetric duopoly games with strategic complements (i) under negative spillovers (NS), if $x^{n}>x_{j}^{s}>$ $x_{i}^{s} \geq x^{c}$, both payoff rankings may arise as follows: $u^{C} \geq u^{F}>$ $u^{L}>u^{N}$ or $u^{F}>u^{C}>u^{L}>u^{N}$, while, for $x^{n}>x_{j}^{s} \geq x^{c}>$ $x_{i}^{s}$, it follows that $u^{F}>u^{C}>u^{L}>u^{N}$. (ii) Under positive spillovers (PS), if $x^{c} \geq x_{i}^{s}>x_{j}^{s}>x^{n}$ both payoff-rankings $u^{C} \geq u^{F}>u^{L}>u^{N}$ or $u^{F}>u^{C}>u^{L}>u^{N}$ may arise, while if $x_{i}^{s}>x^{c} \geq x_{j}^{s}>x^{n}$, the only possible payoff ranking is $u^{F}>u^{C}>u^{L}>u^{N}$.

Proof. It follows straightforwardly by Corollary 2 and Propositions 1,3 , and 5 .

However, as the next example illustrates, in duopoly games with strategic complements (as, for instance, the classical price duopoly game), the case in which the follower's payoff overcomes the collusive payoff remains a rather unusual event. When the follower's best reply is a contraction, it is unlikely that the leader's equilibrium strategy is so low (high) under NS (PS) for the follower to overcome the symmetric collusive payoff. To obtain this, the (increasing) best replies have to be very flat under NS (see Example 4) and very steep under PS.

Example 8 (Bertrand with differentiated products and strategic complements). Let firm $i$ 's $(i=1,2)$ market demand be $q_{i}\left(p_{i}, p_{j}\right)=\left(1-p_{i}+\beta p_{j}\right)^{b}$, where $p_{i}$ and $p_{j}$ denote the prices charged by the two firms. Let also $b>0,0<\beta<1$, and let production costs be normalized to zero for both firms. Payoffs are, therefore, simply given by $u_{i}\left(p_{i}, p_{j}\right)=\left(1-p_{i}+\beta p_{j}\right)^{b} p_{i}$. Note that, for $\beta>0$, these payoffs exhibit increasing differences and best replies are increasing (prices act as strategic complements). Externalities are positive. When the game is played simultaneously and noncooperatively, the equilibrium payoffs are $u^{N}=(b-\beta+1)^{-1-b}(\beta-1+b-\beta+1)^{b}$, while, when the game is played sequentially, the leader obtains

$$
u^{L}=\left(b-\beta^{2}+1\right)^{-1}(b+1)^{-1}(b+\beta+1) \Delta,
$$


for

$$
\begin{gathered}
\Delta=\left(\frac{\beta\left(b \beta^{2}-\beta-b \beta-b^{2}-2 b-1\right)}{(b+1)^{2}\left(\beta^{2}-b-1\right)}\right. \\
\left.-\frac{b+\beta+1}{2 b+b^{2}-\beta^{2}-b \beta^{2}+1}+1\right)^{b},
\end{gathered}
$$

and the follower

$$
u^{F}=(b+1)^{-2}\left(\beta^{2}-b-1\right)^{-1}\left(b \beta^{2}-\beta-b \beta-b^{2}-2 b-1\right) \Gamma
$$

for

$$
\begin{aligned}
\Gamma=( & \frac{\beta(b+\beta+1)}{2 b+b^{2}-\beta^{2}-b \beta^{2}+1} \\
& \left.\quad-\frac{\left(b \beta^{2}-\beta-b \beta-b^{2}-2 b-1\right)}{(b+1)^{2}\left(\beta^{2}-b-1\right)}+1\right)^{b} .
\end{aligned}
$$

Finally, when the two firms decide to merge and act as a monopolist, every firm obtains

$$
u^{C}=((\beta-1)+(b+1)(1-\beta))^{b}((b+1)(1-\beta))^{b-1} .
$$

Simple calculations show that, for all reasonable values of the parameters, the typical payoff ranking is $u^{C}>u^{F}>$ $u^{L}>u^{N}$. Moreover, the following ranking of players' actions (prices) obtains $p^{c}>p_{i}^{s}>p_{j}^{s}>p^{n}$. Figure 5 depicts this case for $\beta=0.5$ and $b=1$. No substantial modifications of the above rankings are obtained by manipulating parameters of the model.

3.2. Games with Strategic Substitutes. When players' actions are strategic substitutes, it can be shown that there are only two possible rankings of equilibrium strategies and payoffs under either negative or positive externalities. These are characterized in the next proposition.

Proposition 9. (i) In all symmetric duopoly games with strategic substitutes, the following rankings between equilibrium strategies can arise: either $x_{i}^{s}>x_{i}^{n}>x_{j}^{s} \geq x^{c}$ or $x_{i}^{s}>$ $x^{n} \geq x^{c} \geq x_{j}^{s}$ under NS and $x_{j}^{s} \geq x^{c} \geq x_{i}^{n}>x_{i}^{s}$ or $x^{c}>x_{j}^{s}>x^{n}>x_{i}^{s}$ under PS, where $x_{j}^{s}=r_{j}\left(x_{i}^{s}\right), i, j=1,2$.

(ii) Moreover, only two alternative payoff rankings are possible: either $u^{C} \geq u^{L}>u^{N}>u^{F}$ or $u^{L}>u^{C} \geq u^{N}>u^{F}$.

Proof. (i) Similarly to Proposition 6 we can write, for player $i$ acting as a leader,

$$
u_{i}\left(x_{i}^{s}, r_{j}\left(x_{i}^{s}\right)\right)>u_{i}\left(x_{i}^{n}, x^{n}\right) \geq u_{i}\left(x_{i}^{s}, x^{n}\right),
$$

where the first inequality stems from the fact that $\mathbf{x}^{s} \neq \mathbf{x}^{n}$ and the second from the property of the Nash equilibrium. Therefore, it follows that $r_{j}\left(x_{i}^{s}\right)=x_{j}^{s}<x^{n}$ under NS and

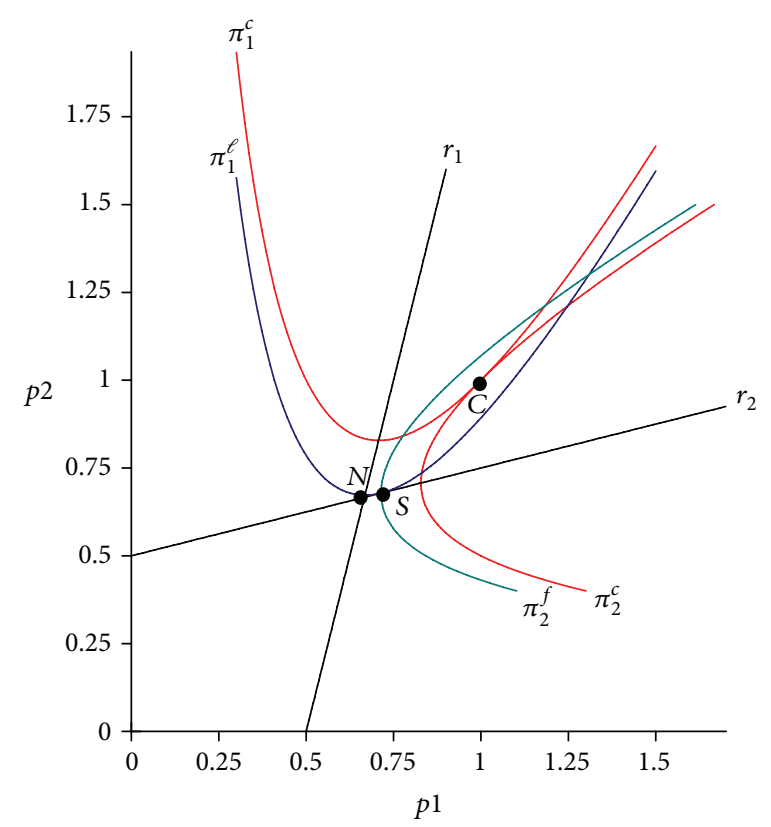

FIGURE 5: Numerical example (price competition with strategic complements)—case $b=1$ and $\beta=0.5$. Red: coop. isoprofits; blue: leader's isoprofit; green: follower's isoprofit.

$r_{j}\left(x_{i}^{s}\right)=x_{j}^{s}>x^{n}$ under PS. Moreover, since actions are strategic substitutes, $x_{i}^{s}>x^{n}$ under NS and $x_{i}^{s}<x^{n}$ under PS, given that both profiles $\mathbf{x}^{s}$ and $\mathbf{x}^{n}$ lie along the follower's (decreasing) best reply. Thus, $x_{i}^{s}>x^{n}>x_{j}^{s}$ under NS and $x_{j}^{s}>x^{n}>x_{i}^{s}$ under PS. By the symmetry and monotonicity of players' payoffs and by the efficiency of the cooperative strategy profile, we obtain also that $x^{c} \leq x_{i}^{n}$ under NS and $x^{c} \geq x_{i}^{n}$ under PS. We remain, therefore, with the following two inequalities: either $x_{i}^{s}>x^{n}>x_{j}^{s} \geq x^{c}$ or $x_{i}^{s}>x^{n}>$ $x^{c} \geq x_{j}^{s}$ under NS, and $x_{j}^{s} \geq x^{c} \geq x^{n}>x_{i}^{s}$ or $x^{c} \geq$ $x_{j}^{s}>x^{n}>x_{i}^{s}$ under PS. Both cases may arise. (ii) From Proposition 1 we know that $u^{C} \geq u^{N}>u^{F}$ when actions are strategic substitutes. Given that $u^{L}>u^{N}$, it follows that only two rankings are possible: either $u^{C} \geq u^{L}>u^{N}>u^{F}$ or $u^{L}>u^{C} \geq u^{N}>u^{F}$, as also the numerical example below illustrates.

Example 10 (Cournot duopoly with strategic substitutes). Let assume an inverse demand function given by $P(Q)=$ $(1-Q)^{b}$, with $Q=\left(q_{1}+q_{2}\right)<1, b>0$ and zero production costs for both firms. The payoffs can be obtained as $u_{i}\left(q_{i}, Q\right)=(1-Q)^{b} q_{i}$, for $i=1,2$. For $b>0$ payoffs exhibit decreasing differences and, therefore, players best replies are decreasing. Spillovers are negative. When the game is played simultaneously à la Nash firms' equilibrium payoffs are given by $u^{N}=b^{b}(b+2)^{-(b+1)}$. When the game is played $\grave{a} l a$ Stackelberg, the leader obtains $u^{L}=b^{2 b}(b+1)^{-(2 b+1)}$ and the follower $u^{F}=b^{(2 b+1)}(b+1)^{-(2 b+2)}$, respectively. Finally, when the two firms collude, they obtain each $u^{C}=b^{b} 2(b+1)^{-(b+1)}$. Simple computations show that for $b \geq 1$ (linear or convex 


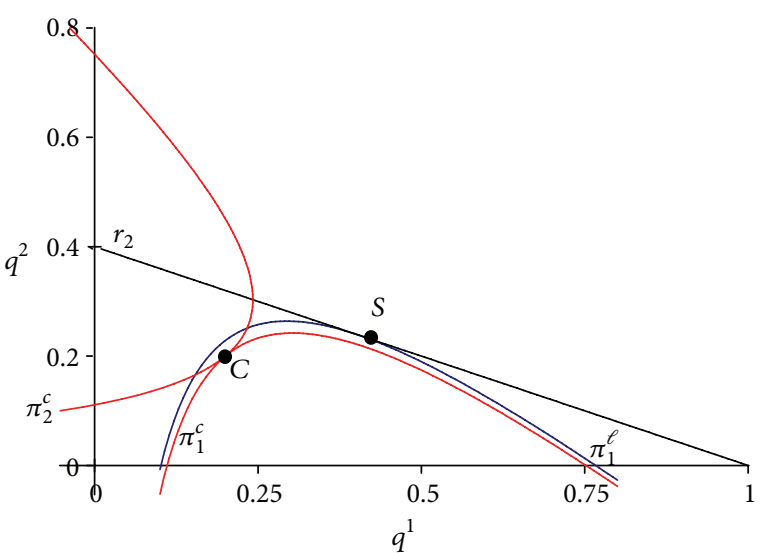

FIGURE 6: Numerical example (Cournot with strategic substitutes) case $b \geq 1$. Red: coop. isoprofits; blue: leader's isoprofit.

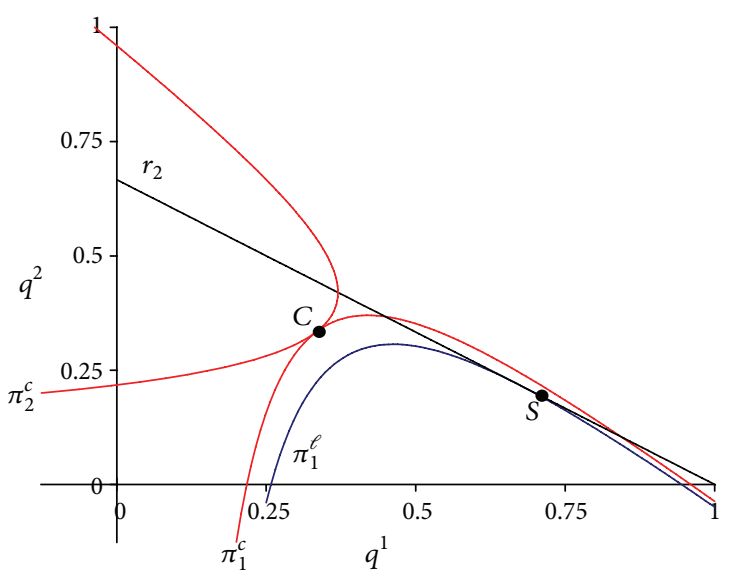

FIGURE 7: Numerical example (Cournot with strategic substitutes)case $0<b<1$. Red: coop. isoprofits; blue: leader's isoprofit.

demand), $u^{C} \geq u^{L}>u^{N}>u^{F}$, with the equal sign holding only for $b=1$. Figure 6 depicts this relation among players' payoffs. When, instead, $0<b<1$ (concave demand), the payoff ranking is the following: $u^{L}>u^{C}>u^{N}>u^{F}$. Figure 7 illustrates this case. Figure 8 shows instead that the ranking $u^{L}>u^{C}$ arises when the inverse demand is strongly concave (which occurs for a very low $b$ ) and that the dominance of leader's payoff over the collusive payoff may occur in both cases in which the follower's strategy is either higher or lower than the collusive strategy.

\section{Endogenous Timing and Collusion}

In order to check some of the potential consequences of our results, we introduce here a simple setting that extends Hamilton and Slutsky's [27] endogenous timing game (known as extended game with observable delay) by including in this game the possibility for players to commit to full cooperation. The game runs as follows. At an initial stage players announce simultaneously their purpose to play early

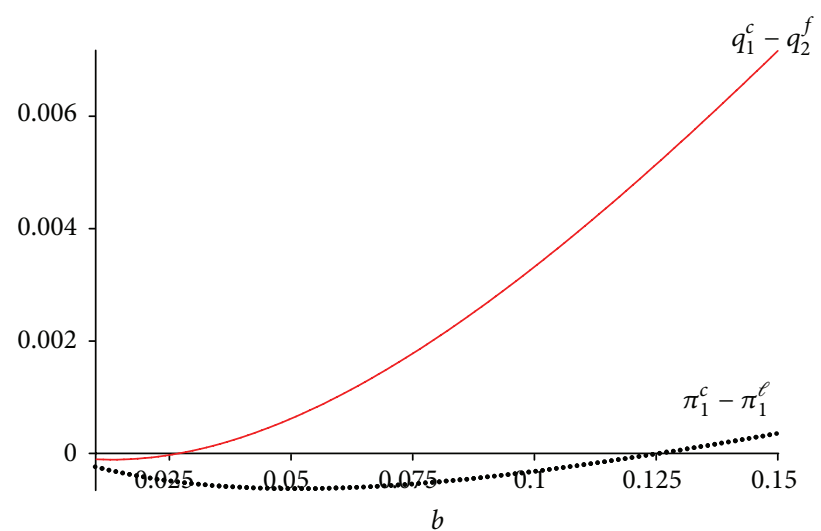

FIGURE 8: Numerical example-red line: $\left(x^{c^{t}}-x_{j}^{s}\right)=$ Coop. output follower's output; black dotted line: $\left(\pi^{c}-\pi^{\ell}\right)=$ coop. payoff - leader's payoff. $b=0.1, \ldots, 0.15$.

or late a duopoly game and their intention to cooperate or not with the rival. The rules of the game are rather simple: when both players announce their intention to cooperate, the agreement is binding and players collude at the selected time. Otherwise, they play independently with the timing as prescribed by their own announcement. This game basically requires the unanimity of players to cooperate. Note that there exist many real life examples in which the willingness of people to take a joint action and the timing of their action are highly complementary, as for example, friends deciding whether to spend or not a holiday together, firms deciding if signing or not a R\&D agreement, and so on. Our aim here is to check for the existence of subgame perfect Nash equilibria (in pure strategies) (SNE) of such cooperation-timing duopoly game.

Formally, the game can be described as follows. We let, at a pre-play stage denoted $t_{0}$, the two players announce simultaneously their intention to cooperate or not with the rival, as well as the timing $\tau=\left(t_{1}, t_{2}\right)$ they intend to play a given duopoly game. Every player's announcement set $A_{i}$, for $i=1,2$ and $j \neq i$, can be defined as

$$
A_{i}=\left[\left(\{i, j\}, t_{1}\right),\left(\{i, j\}, t_{2}\right),\left(\{i\}, t_{1}\right),\left(\{i\}, t_{2}\right)\right],
$$

where the first two announcements express collusive intentions, while the remaining two correspond to the usual (noncooperative) timing choices (early, late) of Hamilton and Slutsky's [27] game. Players' announcement space $A=A_{1} \times$ $A_{2}$ contains 16 different announcement profiles $a \in A$ which, in turn, induce the following set $\mathscr{C}$ of time-cooperation configurations $C(a)$ :

$$
\begin{aligned}
\mathscr{C}(A)=[ & \left(\{1,2\}^{t_{1}}\right),\left(\{1,2\}^{t_{2}}\right),\left(\left\{1^{t_{1}}, 2^{t_{2}}\right\}\right), \\
& \left(\left\{1^{t_{2}}, 2^{t_{1}}\right\}\right),\left(\{1\}^{t_{1}},\{2\}^{t_{1}}\right),\left(\{1\}^{t_{2}},\{2\}^{t_{2}}\right), \\
& \left.\left(\{1\}^{t_{1}},\{2\}^{t_{2}}\right),\left(\{1\}^{t_{2}},\{2\}^{t_{1}}\right)\right] .
\end{aligned}
$$

Differently from Hamilton and Slutsky [27], here the two players are allowed to cooperate and form an alliance either 
at period $t_{1}$ or $t_{2}$ or across periods. Players are allowed to cooperate either during one period or across time, that is, sequencing their cooperative strategy over time. They are not allowed instead to cooperate twice, once in period 1 and once in period 2. We assume that in order to be signed, a binding agreement endowed with a specific timing requires the unanimity of all players. Formally, for $i=1,2, j \neq i$, $k=1,2$ and $\tau=\left(t_{1}, t_{2}\right)$,

$$
\begin{gathered}
C(a)=\left(\left\{i^{\tau_{i}}, j^{\tau_{j}}\right\}\right) \quad \text { iff } a_{i}=\left(\{i, j\}, \tau_{k}\right) \\
\text { for } i=1,2 \text { and } k=1,2, \\
C(a)=\left(\{i\}^{\tau_{h}},\{j\}^{\tau_{l}}\right) \text { otherwise. }
\end{gathered}
$$

The above rule prescribes that, if both players agree to cooperate, they can behave collusively in just one period or alternate their collusive strategy over time. However, if just one player disagrees on cooperation, both players end up playing independently in their own preferred time.

The notion used to define the stability of a given time configuration $C(a)$ is simply the Nash equilibrium of the corresponding announcement profile.

Definition 11 (Nash-stability). A configuration $C(a) \in \mathscr{C}(A)$ is Nash-stable if and only if $C=C\left(a^{*}\right)$, with $a^{*}$ possessing the following property:

$$
u_{i}\left(x\left(C\left(a^{*}\right)\right)\right) \geq u_{i}\left(x\left(C\left(a_{i}^{\prime}, a_{j}^{*}\right)\right)\right),
$$

for any $a_{i}^{\prime} \in A_{i}$, for all $i=1,2$ and $j \neq i$.

When a timing-configuration $C=C\left(a^{*}\right)$ is Nash-stable, this implies, in turn, that the profile $\sigma^{*}=\left(a^{*}, x^{*}\right)$ is a subgame perfect Nash equilibrium (SNE) of the extended game. The only warning is that, in the equilibrium path in which players decide cooperatively, they are assumed to behave as a single maximizing entity. Formally, when the announcement profile induces a collusive behavior, players are assumed to sign a binding agreement. A similar approach is taken in the so called endogenous coalition formation literature (see Ray and Vohra [29, 30], Yi [31], Ray [32], and Marini [33] for surveys.) The next proposition illustrates the main implications of our simple model.

Proposition 12. The endogenous timing-cooperation game played by two symmetric players can give rise to the following cases: (i) under strategic complements, for $u^{C} \geq u^{F}$, the set of stable configurations is given by

$$
\begin{aligned}
\mathscr{C}^{*}=[ & \left(\{1,2\}^{t_{1}}\right),\left(\{1,2\}^{t_{2}}\right),\left(\left\{1^{t_{1}}, 2^{t_{2}}\right\}\right), \\
& \left.\left(\left\{1^{t_{2}}, 2^{t_{1}}\right\}\right),\left(\{1\}^{t_{1}},\{2\}^{t_{2}}\right),\left(\{1\}^{t_{2}},\{2\}^{t_{1}}\right)\right] .
\end{aligned}
$$

(ii) Under strategic complements, for $u^{F}>u^{C}$,

$$
\mathscr{C}^{*}=\left[\left(\{1,2\}^{t_{2}}\right),\left(\{1\}^{t_{1}},\{2\}^{t_{2}}\right),\left(\{1\}^{t_{2}},\{2\}^{t_{1}}\right)\right] .
$$

(iii) Under strategic substitutes, for $u^{C} \geq u^{L}$,

$$
\begin{gathered}
\mathscr{C}^{*}=\left[\left(\{1,2\}^{t_{1}}\right),\left(\{1,2\}^{t_{2}}\right),\left(\left\{1^{t_{1}}, 2^{t_{2}}\right\}\right),\right. \\
\left.\left(\left\{1^{t_{2}}, 2^{t_{1}}\right\}\right),\left(\{1\}^{t_{1}},\{2\}^{t_{1}}\right)\right],
\end{gathered}
$$

and (iv) under strategic substitutes, for $u^{L}>u^{C}$,

$$
\mathscr{C}^{*}=\left[\left(\{1,2\}^{t_{1}}\right),\left(\{1\}^{t_{1}},\{2\}^{t_{1}}\right)\right] .
$$

Proof. (i) When players' actions are strategic complements and $u^{C} \geq u^{F}$, Proposition 1 proved that the following ranking between players' payoffs:

$$
u^{C} \geq u^{F}>u^{L}>u^{N} .
$$

Therefore, any cooperative announcement $a=(\{i, j\}, \tau)$ expressed by both players is a Nash equilibrium of the extended time-cooperation game for any $\tau=\left(t_{1}, t_{2}\right)$, since no player can deviate profitably by announcing either $a_{i}^{\prime}=$ $\left(\{i\}, t_{1}\right)$ or $a_{i}^{\prime \prime}=\left(\{i\}, t_{2}\right)$ inducing, in turn, a duopoly played either simultaneously or sequentially. Therefore, all cooperative configurations $\left(\{i, j\}^{\tau}\right)$ or $\left(\left\{i^{t_{h}}, j^{t_{l}}\right\}\right)$ are supported as SNE of the extended game. The noncooperative sequential configuration $\left(\{i\}^{t_{1}},\{j\}^{t_{2}}\right)$ is also a Nash equilibrium announcement, since, by deviating unilaterally, a player can only induce a noncooperative simultaneous play, obtaining, according to (29), a lower payoff. (ii) When $u^{F}>u^{C}$, the cooperative configurations $\left(\{i, j\}^{t_{1}}\right)$ and $\left(\left\{i^{t_{h}}, j^{t_{l}}\right\}\right)$ are no longer Nash-stable since each player can profitably deviate as a follower by announcing $a_{i}^{\prime \prime}=\left(\{i\}, t_{2}\right)$. Both sequential noncooperative configurations can instead be supported as SNE, since full collusion (at time two) is in this case profitable only for the leader and not for the follower. (iii) When players' actions are strategic substitutes and $u^{C} \geq u^{L}$, by Proposition 1 ,

$$
u^{C} \geq u^{L}>u^{N}>u^{F} .
$$

Here all cooperative agreements are Nash equilibrium announcements for any $\tau=\left(t_{1}, t_{2}\right)$. Also all noncooperative simultaneous configurations $\left(\{i\}^{t_{1}},\{j\}^{t_{1}}\right)$ are stable, since by deviating unilaterally with an announcement $a_{i}^{\prime \prime}=\left(\{i\}, t_{2}\right)$, a player ends up playing sequentially as a follower, receiving a lower payoff. (iv) Finally, when $u^{L}>u^{C}$, the cooperative configurations $\left(\{i, j\}^{t_{2}}\right)$ and $\left(\left\{i^{t_{2}}, j^{t_{2}}\right\}\right)$ are not stable since players can deviate by announcing $a_{i}^{\prime}=\left(\{i\}, t_{1}\right)$ and obtaining a higher payoff as a leader. The simultaneous (noncooperative) configuration played at time 1 is also stable since no player can deviate as a leader, but only as a follower, and the latter deviation is no profitable.

Our simple extension of Hamilton and Slutsky [27] has helped to reach at least one clear-cut conclusion. It is as follows: intertemporal cooperation (or cooperation across time) is, overall, the most vulnerable form of cooperation among players. This is because such type of cooperation may be subject to the objections of players in the role of leaders and followers more frequently than if cooperation takes place just in a single period. 


\section{Concluding Remarks}

This paper has presented a first attempt to connect two usually distinct issues concerning players' strategic interaction: one dealing with their timing of play and the other with their capacity to collude. We have shown that the nature of interaction between players in the strategic setting (duopoly game) plays a crucial role for the decision of players to sign or not a binding agreement and to sequence or not their strategies.

\section{Appendix}

Lemma A.1 (existence of a symmetric cooperative equilibrium). Let players' payoffs be continuous and strictly quasiconcave and their strategy set be compact and convex. Then, the strategy profile $x^{c} \in \arg \max _{\mathbf{x} \in X^{2}} \sum_{i=1,2} u_{i}(\mathbf{x})$ is such that $x_{i}^{c}=x_{j}^{c}$.

Proof. Compactness of each $X$ implies compactness of $X^{2}$. Continuity of each player's payoff $u_{i}(\mathbf{x})$ on $\mathbf{x}$ implies the continuity of the social payoff function $\sum_{i=1,2} u_{i}(\mathbf{x})$. Existence of an efficient profile $x^{c} \in X^{2}$ directly follows. We prove that, under our assumptions, such a strategy profile is symmetric. Suppose $x_{i}^{c} \neq x_{j}^{c}$. By symmetry we can derive from $\mathbf{x}^{c}$ a new vector $\widetilde{\mathbf{x}}$ permuting the strategies of players $i$ and $j$ such that

$$
\sum_{i=1,2} u_{i}(\widetilde{\mathbf{x}})=\sum_{i=1,2} u_{i}\left(\mathbf{x}^{c}\right)
$$

and hence, by the strict quasiconcavity of all $u_{i}(\mathbf{x})$, for all $\delta \epsilon$ $(0,1)$ we have that

$$
\sum_{i=1,2} u_{i}\left(\delta \widetilde{\mathbf{x}}+(1-\delta) \mathbf{x}^{c}\right)>\sum_{i=1,2} u_{i}\left(\mathbf{x}^{c}\right)
$$

Since, by the convexity of $X$, the strategy vector $\left(\delta \widetilde{\mathbf{x}}+(1-\delta) \mathbf{x}^{c}\right) \in X^{2}$, we obtain a contradiction.

\section{Conflict of Interests}

The authors declare that there is no conflict of interests regarding the publication of this paper.

\section{Acknowledgments}

The authors wish to thank Christian Ewerhart, Attila Tasnadi, Jacquelin Morgan, Maria Luisa Petit, George Zaccour, and the participants at the Oligo workshop in Budapest, the MDEF workshop in Urbino, the SING7 conference in Paris, and seminar audience at CREI Roma III and Sapienza University for their useful comments and discussions.

\section{References}

[1] I. Mouraviev and P. Rey, "Collusion and leadership," International Journal of Industrial Organization, vol. 29, no. 6, pp. 705717, 2011.
[2] C. d'Aspremont, A. Jaquemin, J. Gabszewicz, and J. Weymark, "On the stability of dominant cartels," Canadian Journal of Economics, vol. 14, pp. 17-25, 1982.

[3] C. d'Aspremont and J. J. Gabszewicz, "On the stability of collusion," in New Developments in the Analysis of Market Structure, J. Stiglitz and G. F. Mathewson, Eds., MIT Press, Boston, Mass, USA, 1986.

[4] M. Donsimoni, N. Economides, and H. Polemarchakis, "Stable cartels," International Economic Review, vol. 27, pp. 317-327, 1986.

[5] A. Daughety, "Beneficial concentration," American Economic Review, vol. 80, no. 5, pp. 1231-1237, 1990.

[6] D. Levine, "Horizontal mergers: the 50-percent benchmark," American Economic Review, vol. 80, no. 5, pp. 1238-1245, 1990.

[7] S. Huck, K. A. Konrad, and W. Müller, "Big fish eat small fish: on merger in Stackelberg markets," Economics Letters, vol. 73, no. 2, pp. 213-217, 2001.

[8] J. S. Heywood and M. McGinty, "Leading and merging: convex costs, Stackelberg, and the merger paradox," Southern Economic Journal, vol. 74, no. 3, pp. 879-893, 2008.

[9] M. Escrihuela-Villar and R. Faulí-Oller, "Mergers in asymmetric Stackelberg markets," Spanish Economic Review, vol. 10, no. 4, pp. 279-288, 2008.

[10] M. Marini, M. L. Petit, and R. Sestini, "Strategic timing in R\&D agreements," Economics of Innovation and New Technology, 2013.

[11] E. van Damme and S. Hurkens, "Endogenous Stackelberg leadership," Games and Economic Behavior, vol. 28, no. 1, pp. 105-129, 1999.

[12] E. van Damme and S. Hurkens, "Endogenous price leadership," Games and Economic Behavior, vol. 47, no. 2, pp. 404-420, 2004.

[13] B. von Stengel, "Follower payoffs in symmetric duopoly games," Games and Economic Behavior, vol. 69, no. 2, pp. 512-516, 2010.

[14] E. Gal-Or, "First mover and second mover advantages," International Economic Review, vol. 26, pp. 649-653, 1985.

[15] R. Amir, I. Grilo, and J. Jin, "Demand-induced endogenous price leadership," International Game Theory Review, vol. 1, pp. 219-240, 1999.

[16] S. Dowrick, "von Stackelberg and Cournot duopoly: choosing roles," The RAND Journal of Economics, vol. 17, no. 2, pp. 251260, 1986.

[17] R. Amir, "Endogenous timing in two-player games: a counterexample," Games and Economic Behavior, vol. 9, no. 2, pp. 234-237, 1995.

[18] R. Amir and I. Grilo, "Stackelberg versus Cournot equilibrium," Games and Economic Behavior, vol. 26, no. 1, pp. 1-21, 1999.

[19] S. Currarini and M. Marini, "A sequential approach to the characteristic function and the core in games with externalities," in Advances in Economic Design, M. Sertel and A. Kara, Eds., Springer, Berlin, Germany, 2003.

[20] S. Currarini and M. Marini, "A conjectural cooperative equilibrium in strategic form games," in Game Practise and the Environment, C. Carraro and V. Fragnelli, Eds., Kluwer Academic Press, Norwell, Mass, USA, 2004.

[21] B. von Stengel and S. Zamir, "Leadership games with convex strategy sets," Games and Economic Behavior, vol. 69, no. 2, pp. 446-457, 2010.

[22] D. Levine, "Stackelberg, Cournot and collusive monopoly: performance and welfare comparison," Economic Inquiry, vol. 26, pp. 317-330, 1988. 
[23] C. Figuières, M. Tidball, and A. Jean-Marie, "On the effects of conjectures in a symmetric strategic setting," Research in Economics, vol. 58, no. 1, pp. 75-102, 2004.

[24] D. M. Topkis, Supermodularity and Complementarity, Princeton University Press, Princeton, NJ, USA, 1998.

[25] S. W. Salant and G. Shaffer, "Optimal asymmetric strategies in research joint ventures," International Journal of Industrial Organization, vol. 16, no. 2, pp. 195-208, 1998.

[26] D. Leahy and P. J. Neary, "Symmetric research joint ventures: cooperative substitutes and complements," International Journal of Industrial Organization, vol. 23, pp. 5381-6397, 2005.

[27] J. H. Hamilton and S. M. Slutsky, "Endogenous timing in duopoly games: Stackelberg or Cournot equilibria," Games and Economic Behavior, vol. 2, no. 1, pp. 29-46, 1990.

[28] M. Amir, R. Amir, and J. Jin, "Sequencing R\&D decisions in a two-period duopoly with spillovers," Economic Theory, vol. 15, no. 2, pp. 297-317, 2000.

[29] D. Ray and R. Vohra, "Equilibrium binding agreements," Journal of Economic Theory, vol. 73, no. 1, pp. 30-78, 1997.

[30] F. Bloch, "Coalition formation in games with spillovers," in The Endogenous Formation of Economic Coalitions, C. Carraro, Ed., Fondazione Eni Enrico Mattei Series on Economics and the Environment, Elgar, Cheltenham, UK, 2003.

[31] S. S. Yi, "The endogenous formation of economic coalitions: the partition function approach," in The Endogenous Formation of Economic Coalitions, C. Carraro, Ed., Fondazione Eni Enrico Mattei Series on Economics and the Environment, pp. 80-127, Elgar, Cheltenham, UK, 2003.

[32] D. Ray, A Game-Theoretic Perspective on Coalition Formation, Oxford University Press, Oxford, UK, 2007.

[33] M. A. Marini, "Games of coalition and network formation: a survey," in Networks, Dynamics and Topology, A. Naizmada, S. Stefani, and A. Torriero, Eds., Springer, London, UK, 2009. 


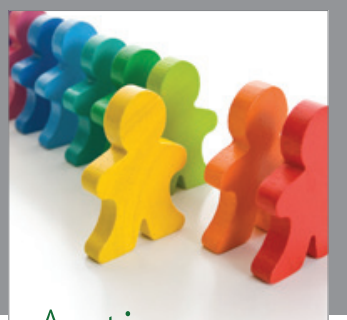

Autism

Research and Treatment
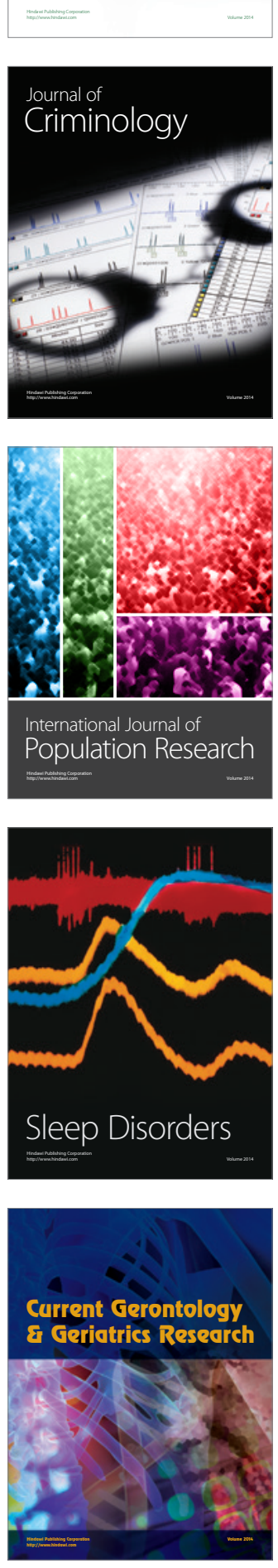
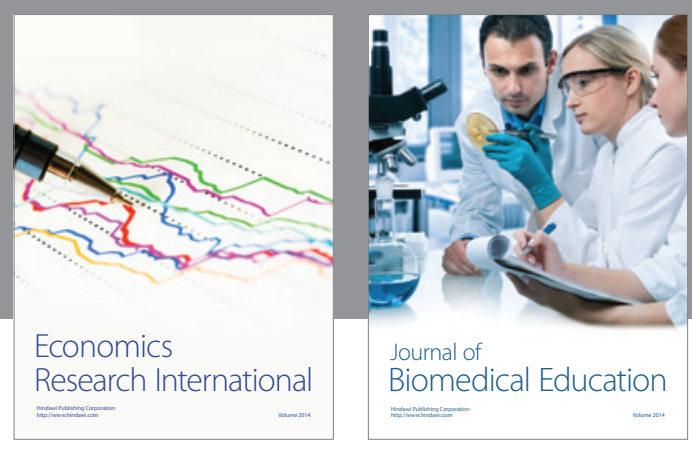

Journal of

Biomedical Education

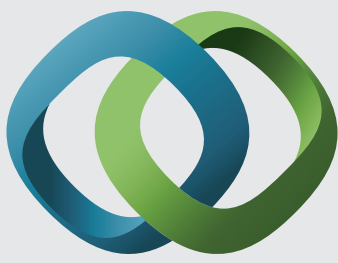

\section{Hindawi}

Submit your manuscripts at

http://www.hindawi.com
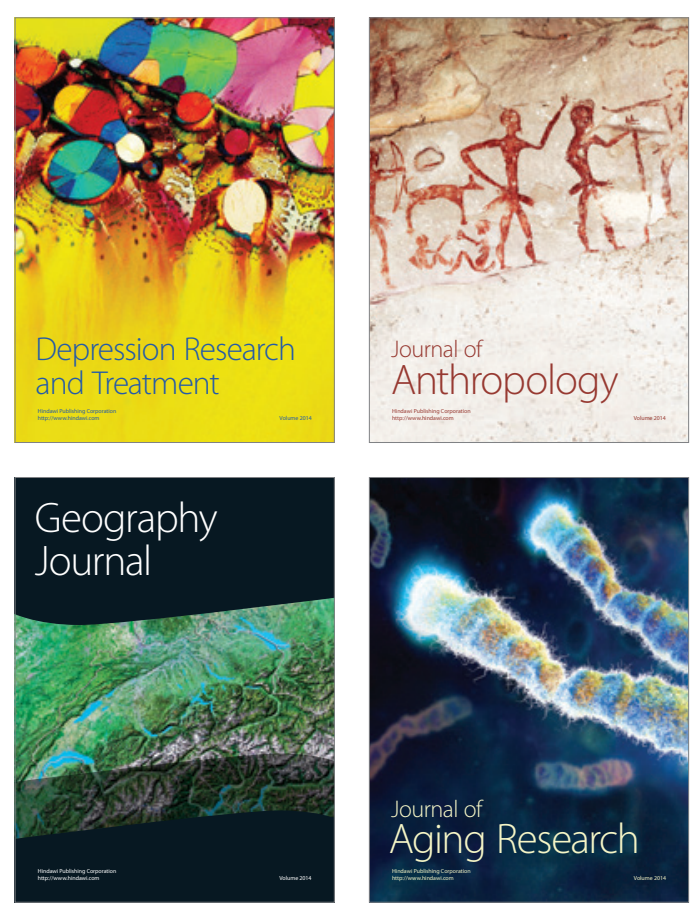

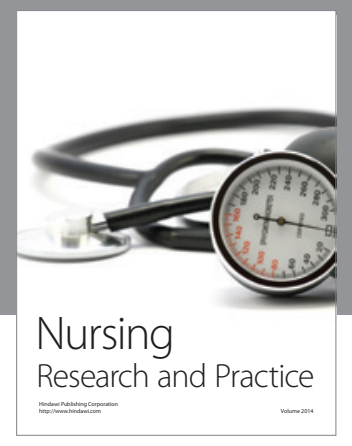

Nursing

Research and Practice

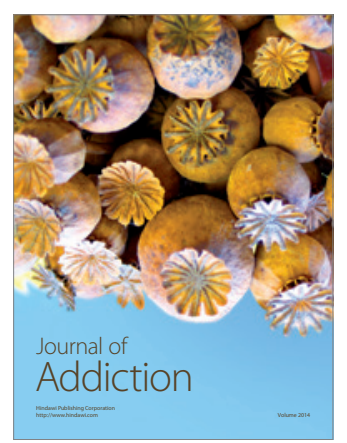

Child Development

Research

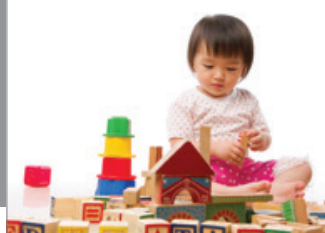

迥
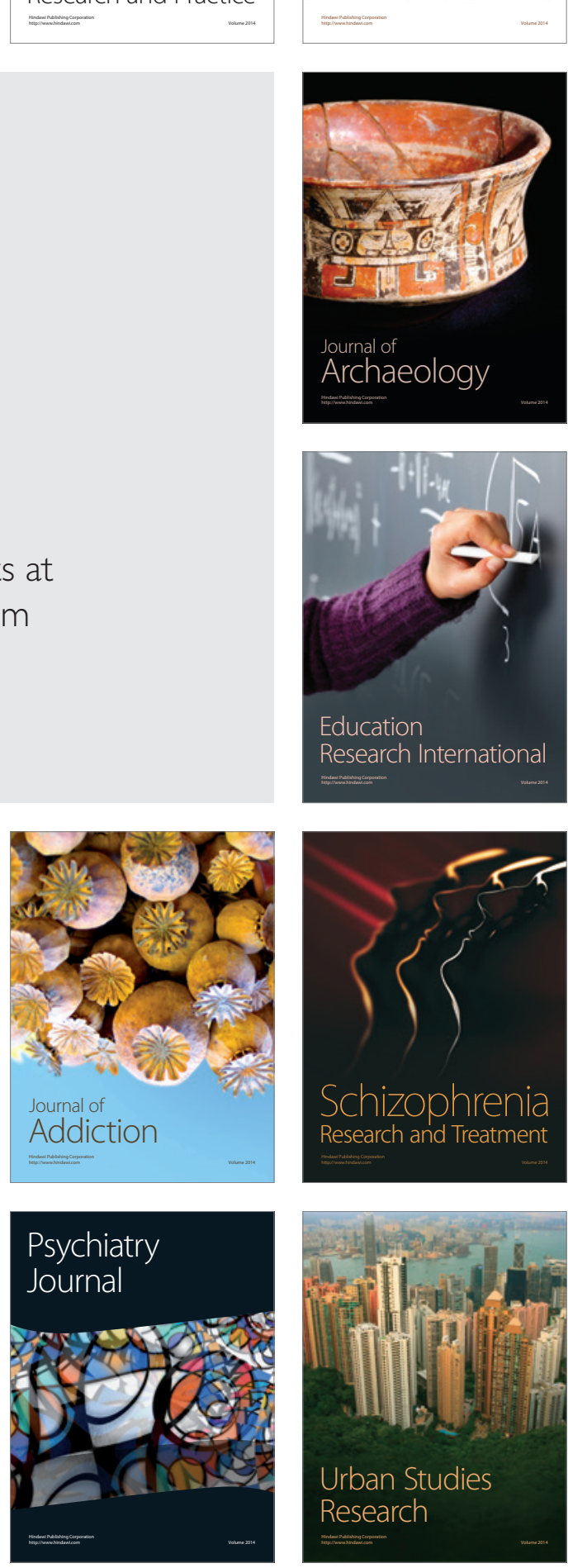